Henry: Untersuchungen über Derivate etc. 231

\title{
Untersuchungen über Derivate des Glycerins von
}

L. Henry.

(Aus dem Bull. de l'acad. de Belg. [2] 37. Nr. 1.)

Veber Diallylabkömmlinge.

1. Oktobromür des Dipropargyls, $\mathrm{C}_{6} \mathrm{H}_{6} \mathrm{Br}_{8}$.

Kürzlich ${ }^{1}$ ) habe ich einen mit Benzol $\mathrm{C}_{6} \mathrm{H}_{6}$ isomeren Kohlenwasserstoff von einer ganz neuen Gattung, das Dipropargyl oder Disllylenyl $\mathrm{C}_{3} \mathrm{H}_{3}-\mathrm{C}_{3} \mathrm{H}_{3}$, kennen gelehrt.

Das Studium dieses Körpers führt zu mancherlei Fragen und wird mich, wie es scheint, lange beschäftigen. Von allen Fragen habe ich zuerst die nach seiner Atomigkeit oder seiner Sättigungscapacität zu beantworten gesucht. Dieser Pankt ist in der That von fundamentaler Wichtigkeit für die Charakteristik und die chemische Constitution dieses mit ungewöhnlichon Eigenschaften begabten Körpers.

Ich habe die Genugthuung, dieses Ziel erreicht zu haben. Nach seiner Entstehung aus dem Diallyl und seiner Formel muss das Dipropargyl achtatomig (octovalent) sein.
$\mathrm{C}_{6} \stackrel{0}{\mathrm{H}}_{14}$
$\mathrm{C}_{6} \stackrel{4}{\mathrm{H}_{20}}$
$\mathrm{C}_{6} \stackrel{\stackrel{8}{\mathrm{H}_{6}}}{\mathrm{C}_{6}}$
$\begin{array}{lllll}\mathrm{C}_{3} & \mathrm{H}_{5} & \mathrm{C}_{2} & \mathrm{H}_{3}\end{array}$
$\begin{array}{llll}\mathrm{C}_{3} & \mathrm{H}_{5} & \stackrel{1}{\mathrm{C}_{3}} & \mathrm{H}_{3}\end{array}$
$\mathrm{C}_{3} \stackrel{0}{\mathrm{H}}_{\mathbf{B}}$
$\mathrm{C}_{3} \stackrel{3}{\mathrm{H}}_{5}$
$\mathrm{C}_{3} \stackrel{5}{\mathrm{H}_{3}}$

Dies führt zu dem Diacetylen- oder vielmehr DialJylencharakter, welchen ich an diesem Kohlenwasserstoff nachgewiesen habe, und welcher seinen Ausdruck in der ihm früher von mir vorläufig gegebenen Strukturformel findet:

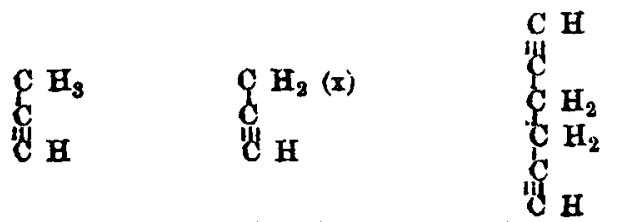

Allylen. Propargylverbindungen. Diproparegl.

2) Dies. Journ. [2] 8, 54. - Bull. de l'acad. de Belg. 86, 41. 
Der Versuch hat diese theoretischen Betrachtungen vollständig bestätigt; es ist $\mathrm{mir}$ in der That gelungen, ein Dipropargyloktobromid darzustellen. Tch habe schon früher zum Theil wenigstens die Einwirkung von Brom auf das Dipropargyl mitgetheilt; dieselbe ist ausserordentlich heftig und energisch und führt unmittelbar zur Bildung eines Tetrabromids, $\mathrm{C}_{6} \mathrm{H}_{6} \mathrm{Br}_{4}$.

Letzteres, eine Flüssigkeit, vermag sich noch mit Brom zu vereinigen; die Einwirkung ist bei gewöhnlicher Temperatur langøam, schnell und ziemlich lebhaft, wenn man das Brom mit gelinde erwärntem Tetrabromür mischt. Das Gemenge wird nach einigen Minuten fest und krystallinisch. Ich brauche wohl nicht hinzuzufügen, dass man Brom in einer Menge anwendet, welche den Formeln $\mathrm{C}_{6} \mathrm{H}_{6} \mathrm{Br}_{4}$ und $\mathrm{Br}_{4}$ entspricht.

Die Reinigung des Products is schüssige Brom wird durch Waschen mit Natronlauge entfernt; die tlüssigen Theile der Substanz werden durch längeres Ausbreiten derselben auf einem porösen Ziegelstein beseitigt. Nach mehrmaligem Umkrystallisiren aus vether ist der Körper vollkommen rein.

Das Dipropargyloktobromid hat ein bemerkenswerthes Aussehen; es scheidet sich beim freiwilligen Verdunsten seiner Lösung in Schwefelkohlenstoff in vollkommen durchsichtigen, wohl ausgebildeten Krystallen ab. Dieselben

Fig. 1.

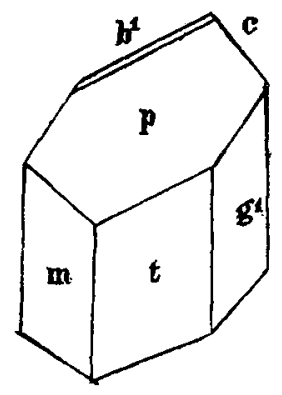

Fig. 2.

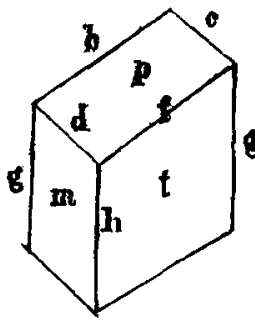
sind farblos, hart und zerbrechlich, brechen stark das Licht. Ich erhielt Krystalle, welche mindestens $1 \mathrm{Cm}$. lang waren. Würde man mit einer grösseren Menge operiren, als mir zur Verfügung stand, so würde man ohne Zweifel solche von grösseren Dimensionen gewinnen. Die Krystalle sind Prismen oder Tafeln. Die oben stehenden Figuren 
zeigen die beiden häufigsten Formen; der Krystall Fig. 2 sielat wie ein Rhomboeder aus. Sie gehören dem triklinischen Systeme an.

Ch. de la Valee-Poussin hat dieselben untersucht.

Die Lüngen und Neigungen der Axen konnten nicht bestimmt werden, da zu deren Berechnung messbare Combinationen nöthig gewesen wären, welche fehlten. Die Flëche $b^{2}$ war zu schmal, so dass ihre Neigungen nicht gemessen werden konnten.

Das Dipropargyloktobromid setzt sich ans ätherischer Lösung bei freiwilliger Verdunstung als körniges, glänzend weisses Krystallpulver ab; diese kleinen Krystalle zeigen unter dem Mikroskop die rhomboedrisehe Form der Fig. 2.

Der Körper ist in Wasser vollkommen unlöslich; absoluter Alkohol löst, selbst kochend, nur geringe Mengen davon, das beste Lösungsmittel ist Schwefelkohlenstoff; welcher denselben, namentlich in der Wärme, reichlich aufnimnt. In Aether ist er weniger löslich.

Er schmilzt zwichen $140^{\prime \prime}-141^{\circ}$ und erstarrt gegen $130^{\circ} \mathrm{l}$ ); er ist nicht (ohne Zersetzung) fiüchtig.

Die Analyse des Products hat folgende Zahlen geliefert.

I 0,1436 Grm. der Substanz gaben 0,2990 Brorasilber (uach der Methode von Carius).

II 0,1965 Grm. derselben Probe lieferten 0,4005 Bromsilber.

III 0,2981 Grm. einer anderen Probe galuen 0,6192 AgBr.

IV 0,3604 Grm. gaben 0,7494 AgBr.

1) Im Folgenden sind die Schmelzpunkte einiger anderer organigcher Bromüre zusammengestellt :

Schmelzpunkt.

Vierfach Bromkohlenstoff (Bolas \& Grove) CBra $91^{\circ}$

Aethylenbromid

Acetylentetrabromid $\mathrm{C}_{2} \mathrm{H}_{4} \mathrm{Br}_{2} \quad 9^{0}$

Allyltribromid (Wurtz)

$\mathrm{C}_{2} \mathrm{H}_{2} \mathrm{Br}_{4} \quad 92^{\circ}$

Crotonylentetrabromid $\mathrm{C}_{3} \mathrm{H}_{5} \mathrm{Br}_{3} \quad 16^{\circ}$ $\mathrm{C}_{4} \mathrm{H}_{6} \mathrm{Br}_{4} 115^{0}-116^{\circ}$ (Henninger, Caventou)

Diallyltotrabromid (Tollen s)

Die Schmelzpankte anderer fonter Bromide eind bisher nicht be stimmt worden. 
234 Henry: Untersuchungen über Derivate

V 0,3374 Grm. gaben 0,7070 AgBr.

VI $0,3220 \mathrm{Grm}$. lieferten $0,6732 \mathrm{AgBr}^{1}$ ).

Diese Zahlen entsprechen folgender procentischen $\mathrm{Zu}$ sammensetzung:

\begin{tabular}{|c|c|c|c|c|c|c|c|c|}
\hline \multicolumn{2}{|c|}{$\mathrm{C}_{6} \mathrm{H}_{8} \mathrm{Br}_{8}$} & Berechne & ; ; & U & III & IV & V & \multirow{2}{*}{$\begin{array}{l}\text { VI } \\
-\end{array}$} \\
\hline $\mathrm{C}_{8}$ & $\begin{array}{r}78 \\
72\end{array}$ & 10,02 & - & - & - & - & - & \\
\hline$H_{8}$ & 6 & 0,83 & - & - & - & - & - & - \\
\hline $\mathrm{Br}_{8}$ & 640 & 89,13 & 88,60 & 88,70 & 88,71 & 88,4 & 89,16 & 88,95 \\
\hline
\end{tabular}

Die niedrigeren Bromüre haben folgende procentische Zusammensetzung :

$\begin{array}{lcc} & \mathrm{C}_{6} \mathrm{H}_{6} \mathrm{Br}_{4} & \mathrm{C}_{6} \mathrm{H}_{6} \mathrm{Br}_{6} \\ \mathrm{C} & 18,09 & 12,90 \\ \mathrm{H} & 1,50 & 1,07 \\ \mathrm{Br} & 80,41 & 86,03\end{array}$

Das Dipropargyl ist mit Benzol isomer. Obwohl dieses sich im Allgemeinen wie ein gesättigter Kohlenwasserstoff verhält, so vermag es doch mit Chlor, Brom und unterchloriger Säure (HO) $\mathrm{Cl}$ Additionsproducte von der allgemeinen Formel $\left(\mathrm{C}_{6} \mathrm{H}_{6}\right) \mathrm{X}_{6} \mathrm{zu}$ bilden. Es ist von Nutzen, einen Augen blick bei dem Hexabromür des Benzols zu verweilen, um es mit dem Oktobromid des Dipropargyls zu vergleichen.

Die beiden Körper sind vollkommen verschieden nach ihrem Aussehen und ihren Eigenschaften. Das Benzolhexabromür $\mathrm{C}_{6} \mathrm{H}_{6} \mathrm{Br}_{6}$ bildet einen krystallinischen Staub, welcher aus kleinen mikroskopischen Nadeln besteht; es ist in Aether sehr löslich, schmilzt bei einer viel höheren Temperatur, über $200^{\circ}$, bei etwa $210^{\circ}$. Das Hexachlorbenzol selbst schmilzt wie man weiss, erst bei $157^{\circ}$ (Zachar. Heys $)^{2}$ ).

Der Unterschied in der Zusammensetzung der Additionsproducte beider isomerer Kohlenwasserstoffe $\mathrm{C}_{6} \mathrm{H}_{6}$, des Benzols und des Dipropargyls, weist auf einen tiefgreifenden Unterschied in der Constitution beider Körper arbitzt.

1) Bei dell Bestimnungen V und VI wurde länger und atärker

2) Zeitschr. f. Chem. 1871, 298. 
hin, speciell in der Struktur ihres Kernes $C_{6}$ : In dem sechsatomigen Benzol bilden die verschiedenen Kohlenstoffatome nach dem jetzt adoptirten Ausdruck eine geschlossene Kette, in dem achtatomigen Dipropargyl dagegen ist dieselbe, wie auch die innere Anordnung sei, offen.

Diesem fundamentalen Unterschiede in der Struktur der isomeren Moleküle $\mathrm{C}_{6} \mathrm{H}_{6}$, entspricht der ganz ungleiche Grad der Additionsfähigkeit: So leicht und energisch die Fixirung des Broms durch Dipropargyl erfolgt, so sehwer vollzieht sie sich beim Benzol.

Sohliesslich bemerke ich, dass das Dipropargyl die erste und einzige achtatomige Verbindung ist, deren Existenz bisher bekannt geworden ist. Weder die äussere Grenze der Wasserstoffentziehung, noch der hohe Grad der Valenz waren erreicht worden. Der bis jetzt dargestellte höchstatomige Kohlenwasserstoff ist das Valylen $\mathrm{C}_{3} \mathrm{H}_{6}$, welches 1865 von $\mathrm{Reboul}^{1}$ ) bei der Untersuchung von Amylderivaten entdeckt wurde. Dasselbe ist sechsatomig und bildet ein krystallisirendes Bromid, $\mathrm{C}_{5} \mathrm{H}_{6} \mathrm{Br}_{2}$. Dieser Kohlenwasserstoff ist noch wenig untersucht.

Unter den einfachen Körpern kennt man keinen, dessen Valenz 6 übersehreitet; auch die Zahl der sechsatomigen Elemente ist höchst beschränkt; man kann zu dieser Gruppe nur Molyldän und Wolfram rechnen. Mit dem Dipropargyl gelangt man demnach zu Verbindungen und Derivaten eines allgemeinen, vollkommen neuen Typus.

2. Tetrabromid des zweifach gebromten Diallyls (Dibromdiallyl).

$$
\begin{gathered}
\text { Diallyl } \\
\mathrm{C}_{3} \mathrm{H}_{5} \\
\mathrm{C}_{3} \mathrm{H}_{5}
\end{gathered}
$$

Tetrabromür

$\mathrm{C}_{3} \mathrm{H}_{5}-\mathrm{Br}_{2}$
Dibromdiallyl

$\mathrm{C}_{3} \mathrm{H}_{4} \mathrm{Br}$

$\mathrm{C}_{3} \mathrm{H}_{4} \mathrm{Br}$.

Tetrabromür

$\mathrm{C}_{3} \mathrm{H}_{4} \mathrm{Br}-\mathrm{Br}_{2}$

$\mathrm{C}_{3} \mathrm{H}_{4} \mathrm{Br}-\mathrm{Br}_{2}$.

1) Compt. rend. 60, 803. 
236 Henry: Untersachungen über Derivate etc.

Das Dibromdiallyl $\mathrm{C}_{6} \mathrm{H}_{8} \mathrm{Br}_{2}$ vereinigt sich lebhaft mit Brom, wie das Diallyl selbst, doch weniger energisch, zu einem Tetrabromid $\left(\mathrm{C}_{6} \mathrm{H}_{8} \mathrm{Br}_{2}\right) \mathrm{Br}_{4}$.

Dieses Product, anfangs eine dicke, sehr zähe Flüssigkeit, erstarrt mit der Zeit zu einem Krystallbrei. Von den flüssigen Theilen wird derselbe durch längeres Ausbreiten in dünner Schicht auf einem porösen Ziegelstein befreit. Man krystallisirt aus Alkohol um. So dargestellt bildet das Dibromdiallyltetrabronid völlig weisse, perlnutterglänzende weiche Blättchen, welche zwischen $76^{\circ}$ und $77^{\circ}$ schmelzen. Es löst sich leicht in Aether und Alkohol, besonders in heissem, auf. Von dem Diallyltetrabromid unterseheidet dasselbe sich äusserlich; jenes schmilzt übrigens bei $63^{\circ}$ ( Tollens) ${ }^{1}$ ).

Die Analyse des Körpers hat zu folgenden Resultaten geführt:

I. 0,1104 Grm. lieferten 0,2228 Grm. Ag Br (nach Cariu s Methode).

II. 0,1002 Grm. gaben 0,1998 Grm. Ag Br.

III. 0,1008 Grm. gaben 0,2002 Grm. Ag Br.

Dies entspricht folgenden procentischen Werthen

\begin{tabular}{lrrrrr}
\multicolumn{3}{c}{ Berechnet: } & \multicolumn{3}{c}{ Gefunden: } \\
\cline { 2 - 6 } $\mathrm{C}_{6} \mathrm{H}_{8} \mathrm{Br}_{6}$ & & $\mathrm{I}$. & II. & III. \\
$\mathrm{C}_{8}$ & 72 & 12,86 & - & - & - \\
$\mathrm{H}_{8}$ & 8 & 1,43 & - & - & - \\
$\mathrm{Br}_{6}$ & 480 & 85,71 & 85,87 & 85,02 & 85,78
\end{tabular}

Ich beabsichtige, demnächst die Einwirkung der Halogenwasserstoffsäuren auf Dipropargyl z's untersuchen.

Schliesslich möchte ich auf die Beziehungen hinweissen, welche man zwischen Diallyl und Dipropargyl und zwischen gewissen mehrbasischen Säuren, nämlich der Tricarballylund der Aconitsäure $\left(\mathrm{C}_{6} \mathrm{H}_{8} \mathrm{O}_{6}\right.$ und $\left.\mathrm{C}_{6} \mathrm{H}_{6} \mathrm{O}_{6}\right)$ aufzustellen berechtigt ist.

Nach ihrer Entstehungsweise aus dem Tribromhydrin des Glycerins kann man mit Sicherheit der Tricarballylsäure folgende Strukturformel beilegen:

ग) Tollens \& Wagnex. Ber. Berl. Chem. Ges. 6, 588. 
Petersen: Zur Kenntniss der triklinen Foldspatbe. 237

$$
\begin{aligned}
& \begin{array}{lll}
\mathrm{C}_{6} \mathrm{H}_{8} \mathrm{O}_{6} & \left.\left(\mathrm{C}_{3} \mathrm{H}_{5}\right)-(\mathrm{COOH})_{3}\right) & \begin{array}{l}
\stackrel{\mathrm{CH}_{2}}{\mathrm{CH}}-\mathrm{COOH} \\
\mathrm{CH}_{2}-\mathrm{COOH}
\end{array}
\end{array} \\
& \begin{array}{ll}
\text { Tribromhydrin } \mathrm{C}_{3} \mathrm{H}_{5}-\mathrm{Br}_{3} & \begin{array}{l}
\mathrm{CH}_{2} \mathrm{Br} \\
\mathrm{CH} \mathrm{Br}
\end{array} \\
\mathrm{CH}_{2} \mathrm{Br} .
\end{array}
\end{aligned}
$$

Die Constitution der Aconitsäure kann aller Wahrscheinlichkeit nach duroh folgende Formel ausgedrückt werden:

$$
\begin{array}{lll}
\mathrm{C}_{6} \mathrm{H}_{6} \mathrm{O}_{6} & \mathrm{C}_{3} \mathrm{H}_{3}-(\mathrm{COOH})_{3} & \mathrm{CH}_{2}-\mathrm{COOH} \\
& & \stackrel{\mathrm{C}}{\mathrm{C}}-\mathrm{CO}-\mathrm{COOH}
\end{array}
$$

Verhält sich dies so, dann kann man erwarten, dass durch Elektrolyse der resp. Alkalisalze die Tricarballylsäure Diallyl die Aconitsäure ${ }^{1}$ ) Dipropargyl liefern werde.

Man darf ebenso hoffen, mit Hülfe des Propargyltribromürs $\left(\mathrm{C}_{3} \mathrm{H}_{3}\right) \mathrm{Br}-\mathrm{Br}_{2}$ und von Cyankalium die Synthese der Aconitsăure ausführen zu können, wie die der Tricarballylsäure aus dem Allyltribromid $\left(\mathrm{C}_{3} \mathrm{H}_{5}\right) \mathrm{Br}-\mathrm{Br}_{2}$ gelungen ist.

Ich gedenke diese versehiedenen Reactionen zu versuchen, sobald meine mich jetzt beschäftigenden Arbeiten weit genug fortgeschritten sein werden.

\section{Zur Kenntniss der triklinen Feldspatbe;}

\section{Theodor Petersen.}

Gelegentlich meiner Untersuchungen über die Grünsteine $\left.{ }^{2}\right)$ habe ich anoh Veranlassung genommen, mich über die in neuerer Zeit so häufig discutirte Zusammensetzung der Feldspathe auszusprechen und auseinandergesetzt, dass gewichtige Gründe gegen die Annahme der SartoriusTschermak'sohen Mischungstheorie vorliegen.

1) Berthelot hat bekanntlich durch die Hektrolyse einer alkalischen Lösung von aloonitsaurem Kali Acetylen erhalten. Diese Thatsache ormuthigt mich, die Reaction unter anderen Bedingungen zu tudiren. (Bull. Soc. Chin. 9, 103, 1868.)

) Dies. Journ. 1872. 197. 